\title{
Glukokortikoid-Nasensprays bald rezeptfrei?
}

$\mathrm{D}$ er Sachverständigen-Ausschuss für Verschreibungspflicht hat sich mehrheitlich dafür ausgesprochen, Nasensprays, die Beclometason, Flutica-

son, Mometason oder deren Ester enthalten, für die Selbstmedikation freizugeben. Bislang ist nur Beclometason zur nasalen Anwendung rezeptfrei erhält-

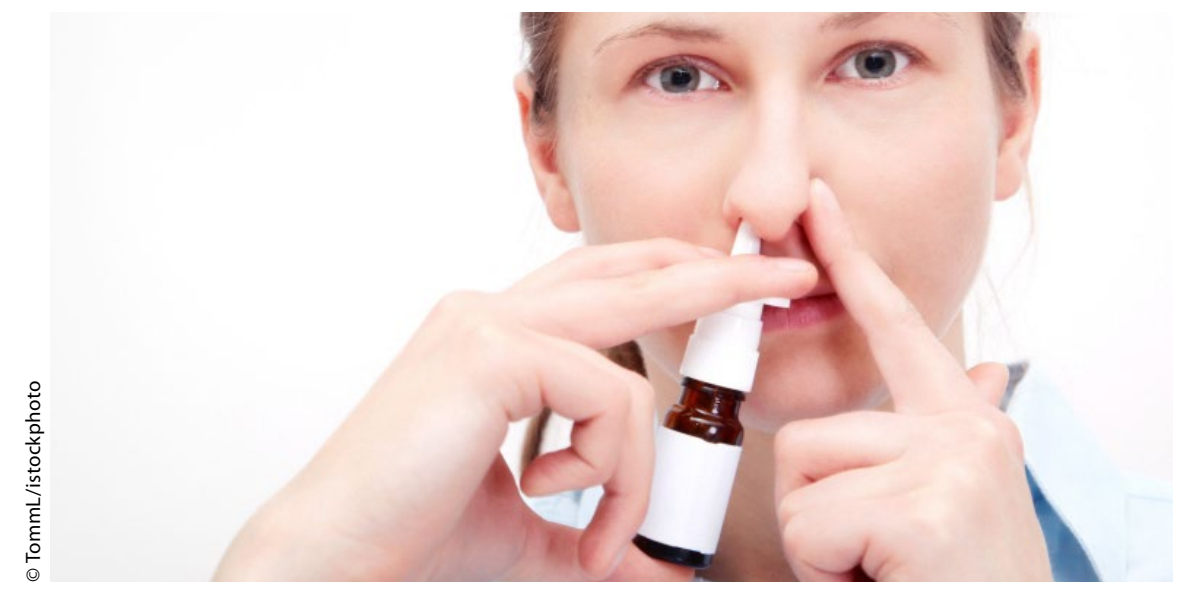

\section{Allergenchip zur Diagnostik wieder verfügbar}

D er Allergenchip ImmunoCAP ${ }^{\circledast}$ ISAC sIgE 112 von Phadia/Thermo Fisher Scientific wird ab sofort wieder produziert und ausgeliefert. Der Allergenchip war im Oktober 2014 vom Markt genommen worden, da ein Verdacht auf falsch positive Testresultate bei 29 der 112 Allergenkomponenten vorlag. Wie der Hersteller nun mitteilte, konnten die Probleme auf verstärkte Hinter- grundsignale bei einem Teil der Allergenkomponenten auf Chips zurückgeführt werden, die zwischen Juni und August 2014 an Labors ausgeliefert wurden. Durch die Optimierung des Kopplungsverfahrens und geringfügige Änderungen im Chipdesign seien die Probleme gelöst und zusätzlich weitere Qualitätskontrollschritte implementiert worden, so das Unternehmen weiter. lich, obwohl alle drei Glukokortikoide ähnlich wirken. Voraussetzungen für die rezeptfreie Abgabe werden sein, dass die Produkte für die Indikation „symptomatische Behandlung der saisonalen allergischen Rhinitis“ zugelassen sind, dass die Erstdiagnose durch einen Arzt gestellt wurde und dass die Anwendung auf Erwachsene beschränkt ist.

Folgt das Bundegesundheitsministerium der Empfehlung, wird die Änderungsverordnung voraussichtlich Ende Juni im Bundesgesetzblatt publiziert. Um den pharmazeutischen Firmen ausreichend Zeit für die Änderung der Verpackungen und Produktinformationen zu geben, wird ihnen in der Änderungsverordnung meist eine Übergangsfrist von drei Monaten eingeräumt. Somit dürfte es etwa bis Ende 2016/Anfang 2017 dauern, bis die drei Glukokortkoidsprays in der Sichtwahl der Apotheken verfügbar sind.

red

Um zu bestätigen, dass der Allergenchip wieder allen Anforderungen gerecht wird, wurde eine externe Validierungsstudie an 23 Zentren in zwölf Ländern durchgeführt. Diese Studie ergab eine gute Reproduzierbarkeit und keine falsch positiven Testergebnisse mit dem Allergenchip. Um eine volle Verfügbarkeit sicherzustellen, hat Thermo Fisher Scientific die durchschnittlichen Lagerbestände erhöht.

red

\section{Nahrungsmittelallergiker auf Reisen}

D as Europäische Verbraucherzentrum (EVZ) Deutschland in Kiel hat für 21 europäische Amtssprachen sowie Türkisch, Russisch, Isländisch und Norwegisch ein Wörterbuch mit Übersetzungen der Namen von 130 allergieauslösenden Lebensmitteln erstellt. Das Wörterbuch hilft weiter, wenn allergiekranke Patienten im Restaurant oder beim Einkauf in einer fremden Sprache verständlich machen müssen, dass Sie auf bestimmte Nahrungsmittel verzichten müssen.
Übersetzt werden beispielsweise Begriffe wie Avocado, Dinkel, Sellerie, Krebstier oder Macadamianuss. Auf einer Karte „Ich habe eine Allergie gegen ..." können die Verbraucher ihre Allergien schon vor der Reise eintragen und dann einfach im Portemonnaie mit sich tragen.

Die Wörterlisten können von der EVZ-Website kostenlos heruntergeladen werden (www.eu-verbraucher.de/ de/verbraucherthemen/reisen-in-der-eu/ vor-der-reise/allergie-woerterbuch). red

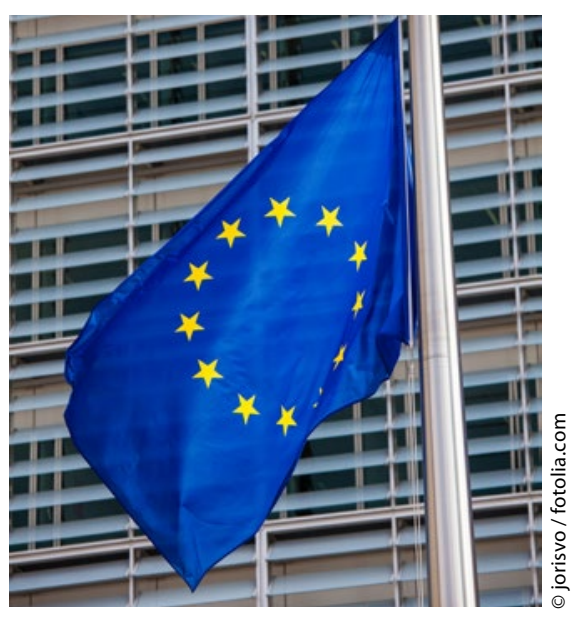

